\title{
Physics of Ferroelastic Crystals. The 6th (11th) International Seminar
}

\author{
L. N. Korotkov ${ }^{a}$ and A. S. Sigov \\ ${ }^{a}$ Voronezh State Technical University \\ ${ }^{b}$ Moscow State University of Radio Engineering, Electronics and Automation (Technical University) \\ DOI: $10.3103 / \mathrm{S} 1062873810090017$
}

The works presented at the seminar were distributed among the following topics: "Phase Transitions, Lattice Dynamics and Soft Modes"; "Structure and Growth of Crystals"; "Thin Films"; "Polar Polymers"; "Heterogeneous, Vitreous, and Inadequate Structures"; "Domains and Domain Boundaries and Their Dynamics"; "Crystalline Structure and Physical Properties"; and "Multiferroics."

A general analysis of the presented papers revealed researchers' steadily growing interest in the physical phenomena of multiferroics, as was reflected in the experimental and theoretical studies presented at the seminar. The fundamental problems related to magnetic, electric, and elastic interactions in condensed homogeneous and heterogeneous structures were the subject of an active discussion within the specially organized section on multiferroics and during round table discussions. Along with this, increased attention was given to the problem of disordered ferroelastics. Scientific interest has shifted lately from quasi-homogeneous objects (e.g., relaxor ferroelectrics) to microand nanostructured composite materials. A great number of experimental studies devoted to investigating different properties of heterogeneous media were presented.

At the same time, we should note the absence of general approaches to describing the physical phenomena in ferroics with micro- and nanosize inhomogeneities, ferroelectric superlattices included. This shows that the problem of nanostructured ferroelastics and ferroelectrics is at the initial stage of its development, both in Russia and beyond it.

A great number of studies have traditionally been devoted to lattice dynamics and structural phase transitions in different classes of ferroelectrics. The experimental results were most often interpreted using the methods of phenomenological theory. At the present stage, they are most well-developed and allow satisfactory description of the most important physical properties of ferroelastics and ferroelectrics.

A remarkable number of reports were devoted to the problems of domain wall dynamics in crystals; nucleation; the interactions of domain boundaries and flaws; acoustic and optical waves; and so on. Special attention was paid to the results of investigations dealing with the formation of a domain structure in substantially disordered materials of the lead magnoniobate-type and solid solutions based on it.

Interest in thin-film ferroelastics and ferroelectrics (particularly in the peculiarities of their shifts and the formation of domain structures in them) can be attributed to both scientific and practical interest. The latter is related to the new possibilities for using thin-film ferroelectrics in NVRAM devices, and for obtaining specified domain (nanodomain) configurations that can find practical application as components of engineering processes in domain engineering.

Finally, let us note that the problems featured at the seminar went beyond "pure" physics of ferroelastics. Their integration with problems related to other fields of science, particularly the physics of semiconductors, polymers, ferromagnetic, and so on, can be clearly traced.

Another trend in the development of the physics of ferroelastics is the intensification of research in the field of active heterogeneous media. This is likely attributable to the challenging potentialities of their practical application.

Some of the seminar participants' papers will be published in the journal Ferroelectrics (2010), while the rest will be presented in this journal and in Crystallography. 